\title{
Detectability of tensor modes in the presence of foregrounds
}

\author{
Mihail Amarie, ${ }^{1, *}$ Christopher Hirata, ${ }^{1}$ and Uroš Seljak ${ }^{1,2}$ \\ ${ }^{1}$ Department of Physics, Jadwin Hall, Princeton University, Princeton, New Jersey 08544, USA \\ ${ }^{2}$ International Center for Theoretical Physics, Strada Costiera 11, 34014 Trieste, Italy
}

(Received 15 August 2005; published 22 December 2005)

\begin{abstract}
In inflationary models gravitational waves are produced in the early universe and generate $B$-type polarization in the cosmic microwave background (CMB). Since $B$ polarization is only generated by gravity waves it does not suffer from the usual cosmic variance. A perfect decomposition of the CMB into $B$-modes and $E$-modes would require data from the entire sky, which in practice is not possible because of the foreground contaminants. This leads to mixing of $E$ polarization into $B$, which introduces cosmic variance contamination of $B$ polarization and reduces sensitivity to gravity wave amplitude even in absence of detector noise. We present numerical results for the uncertainty in the tensor-to-scalar ratio using the Fisher matrix formalism for various resolutions and considering several cuts of the sky, using the foreground model based on dust maps and assuming $90 \mathrm{GHz}$ operating frequency. We find that the usual scaling $\triangle\left(\frac{T}{S}\right) \propto f_{\text {sky }}^{-1 / 2}$ is significantly degraded and becomes $\triangle\left(\frac{T}{S}\right) \propto f_{\text {sky }}^{-2}$ for $f_{\text {sky }}>0.7$. This dependence is affected only weakly by the choice of sky cuts. To put this into a context of what is required level of foreground cleaning, to achieve a $T / S=10^{-3}$ detection at $3 \sigma$ one needs to observe $15 \%$ of the sky as opposed to naive expectation of $0.3 \%$. To prevent contamination over this large sky area at required level one must be able to remove polarized dust emission at or better than $0.1 \%$ of unpolarized intensity, assuming the cleanest part of the sky has been chosen. To achieve $T / S=10^{-4}$ detection at $3 \sigma$ one needs to observe $70 \%$ of the sky, which is only possible if dust emission is removed everywhere over this region at $0.01 \%$ level. Reaching $T / S=10^{-2}$ should be easier: $1 \%$ of the sky is needed over which polarized emission needs to be removed at $1 \%$ of unpolarized intensity if the cleanest region is chosen. These results suggest that foreground contamination may make it difficult to achieve levels below $T / S=10^{-3}$.
\end{abstract}

DOI: 10.1103/PhysRevD.72.123006

PACS numbers: $98.70 . \mathrm{Vc}, 98.80 . \mathrm{Bp}, 98.80 . \mathrm{Cq}$

\section{INTRODUCTION}

The polarization in the cosmic microwave background (CMB) is generated by Thomson scattering of the CMB photons off free electrons. Thomson scattering generates only linear polarization and the polarization can be expressed in terms of the coordinate dependent Stokes parameters $Q$ and $U$. It can be also decomposed in the coordinate independent components in the harmonic space: scalar components denoted by $E$, and pseudoscalar components $B$. For spin $l$, the parity of the $E$ modes is $(-1)^{l}$, while the parity of the $B$ modes is $-(-1)^{l}$. The primordial density perturbations are scalar only, and because of the parity invariance, they can only generate $E$ modes. The gravitational waves present during the inflation are tensor perturbations, so they can generate both $E$ and $B$ modes [1-3]. If the amplitude of the gravity waves is very small relative to scalars it cannot be isolated from the temperature anisotropies or $E$ polarization due to cosmic variance. The $B$ polarization is insensitive to the cosmic variance from the $E$ modes, being affected only by the foregrounds and the instrument noise. The amplitude of the $B$ modes depends on the amplitude of the gravity waves generated during the inflation, which in turn depends on the (as yet unknown) energy scale at which the inflation occurred. The tensor-to-scalar power ratio is often defined as $T / S \equiv C_{2}^{T T \text {,tensor }} / C_{2}^{T T, \text { scalar }}$, i.e. the ratio of the contribu-

*Electronic address: mamarie@princeton.edu tions to the CMB temperature quadrupole. In terms of the energy density during the inflation it can be written as $T / S \sim V_{*} /\left(3.7 \times 10^{16} \mathrm{GeV}\right)^{4}$, where $V_{*}$ is the energy scale of inflation. The possibility of detecting direct evidence of gravity waves from inflation via $B$ modes is currently being considered by a number of ground, balloon, and space based experiments. Notably, a future satellite mission dedicated to $B$-type polarization has been identified as one of the NASA beyond Einstein missions, the inflation probe.

There are a number of outstanding issues that need to be resolved for such a mission to become a reality. Some of these are the question of required sensitivity and angular resolution to achieve the desired energy scale of inflation below $10^{16} \mathrm{GeV}$ [4] and the issue of $B$-mode contamination from gravitational lensing [5]. One of the main issues related to the feasibility of such observation of the CMB polarization is the contamination by foregrounds. The dust present in the interstellar medium (ISM) is one of the main foreground contaminants. It has a thermal vibrational emission spectrum, which is increasing with the frequency in the range of interest for us, and it also shows an increased emission in the $10-90 \mathrm{GHz}$ range, which could be caused by the rotation of small dust particles [6]. The ISM can also be a significant source of synchrotron radiation, which shows a red frequency dependence [7]. The ISM contaminants are present in our galaxy, so the most affected region on a map would be the galactic plane. 
All these contaminants lead to a more realistic scenario in which we have to exclude portions of the sky because of their contamination by foregrounds. This can be done by simply removing some portions of the sky where the contamination is largest, or can be more sophisticated by marginalizing over some foreground templates $[8,9]$. For simplicity, in this paper we only consider the first case. We expect that our conclusion holds qualitatively also for other more sophisticated types of analysis and discusses what are required conditions when this is likely to be valid. Most CMB parameter forecasting studies have assumed that if only a fraction of the sky $f_{\text {sky }}$ is observed then the uncertainties in all parameters is reduced by $f_{\text {sky }}^{-1 / 2}$. However, in case of polarization this ignores an important issue. On the whole sky, the $E$ and the $B$ modes are separable. This statement does not hold anymore on a partial sky as the boundaries of the cuts generate mixing between these modes. In simple geometries one can isolate pure $E$ modes, pure $B$ modes, and also mixed modes for which one cannot discern the $E$ or $B$ nature [10,11]. Pure $E$ modes and mixed modes are contaminated by $E$-mode polarization from scalar perturbations, which are larger than the $B$ modes from gravity waves even at the present upper bound on $T / S$. Thus for these modes some of the same cosmic variance issues arise as for $E$ polarization and temperature anisotropies. As a result the scaling with $f_{\text {sky }}$ can become much worse than expected. A few studies of tensor $B$ modes have taken this issue into account in specific cases such as the WMAP Kp0 cut [10] or cuts based on galactic latitude [12,13], but the full dependence on the sky cut has not been investigated.

Analytic decompositions into $E, B$, and mixed modes, emphasized in previous work, are possible only in a few cases of simple geometry. In a more general case with pixelization effects included, all modes are mixed, but the level of mixing varies so that the $E$-mode contamination ranges from negligible to nearly complete. Qualitatively, the larger the area of the sky coverage and the smaller the length of the boundaries, the smaller the contamination and mode mixing, but analytic results are difficult to obtain. Fortunately the decomposition into pure and mixed states, while useful for heuristic interpretation, is not necessary in the actual analysis of the data. One simply needs to perform the usual likelihood analysis of the data expressed with measured Stokes $Q$ and $U$ parameters. The likelihood analysis is an optimal analysis and as such cannot be improved upon by performing the decomposition into pure and mixed modes.

Broadly speaking there are two contributions to $B$-mode polarization. One is from the recombination epoch (which defines the last scattering surface), which leads to a peak in the power spectrum at $l \sim 100$. The second is from the reionization, which rescatters the $\mathrm{CMB}$ quadrupole on the horizon scale during reionization and leads to a large signal at large angular scales, $l<20$, while at the same time suppressing the recombination signal at smaller scales [14]. If the optical depth to reionization is high, as suggested by WMAP first year results [15-18], then the latter signal dominates in the determination of the gravity wave amplitude [4]. Because of its larger angular scale, this component of the signal also suffers more from $E$-mode mixing into $B$ modes due to incomplete sky coverage. In this paper we include both, varying the amplitude of optical depth over the allowed range.

\section{QUADRATIC ESTIMATOR AND FISHER MATRIX}

We consider the quadratic estimator for the vector $x=$ $\left(Q_{1}, Q_{2}, \ldots Q_{N}, U_{1}, U_{2}, \ldots U_{N}\right)$, where $N$ is the total number of pixels considered and $Q$ and $U$ are the coordinate dependent Stokes parameters. The covariance matrix $C=$ $\left\langle x x^{\dagger}\right\rangle$ is written as

$$
C_{i j}=C_{0, i j}+\frac{T}{S} C_{T / S, i j}
$$

Here $\mathbf{C}_{0}$ is the covariance matrix including noise and scalar fluctuations but no tensors, $T / S$ is the tensor-to-scalar ratio, and $\mathbf{C}_{T / S}$ is the $B$-mode power spectrum template,

$$
C_{T / S, i j}=\sum_{l m} \frac{\partial C_{l}^{B B}}{\partial(T / S)} \mathbf{Y}_{l m}^{B *}\left(\mathbf{n}_{i}\right) \mathbf{Y}_{l m}^{B}\left(\mathbf{n}_{j}\right)
$$

We have left out the $E$-mode power spectrum from the tensors, which contributes negligibly at small $T / S$ due to cosmic variance. In our case, the $\mathbf{C}_{0}$ matrix contains both the contribution from the detector noise and the $E$-mode contribution to the polarization. We work in the limit of $T / S=0$, so we do not consider the second term for the covariance matrix. The quadratic estimator determines the tensor-to-scalar ratio using the relation

$$
\begin{aligned}
\widehat{T / S} & =\left[F^{-1}\right] q, \\
q & =\frac{1}{2} \mathbf{x}^{\dagger} \mathbf{C}_{0}^{-1} \mathbf{C}_{T / S} \mathbf{C}_{0}^{-1} \mathbf{x}-\frac{1}{2} \operatorname{Tr}\left(\mathbf{C}_{0}^{-1} \mathbf{C}_{T / S}\right), \\
F & =\frac{1}{2} \operatorname{Tr}\left(\mathbf{C}_{0}^{-1} \mathbf{C}_{T / S} \mathbf{C}_{0}^{-1} \mathbf{C}_{T / S}\right) ;
\end{aligned}
$$

here $F$ is the $1 \times 1$ Fisher matrix and $\mathbf{C}_{0}$ can be any positive definite Hermitian matrix. In our case the Fisher matrix is reduced to only 1 element, since we have only 1 parameter $(T / S)$. For any choice of $\mathbf{C}_{0}$ the estimator will be unbiased, however the quadratic estimator method has optimum efficiency if $\mathbf{C}_{0}$ is the covariance matrix itself. In our particular case $\mathbf{C}_{0}$ contains the instrument noise which is uncorrelated and also contains the contribution of the scalar modes to the power spectrum. As we are interested in estimating $\triangle\left(\frac{T}{S}\right)$, we want to evaluate the Fisher matrix element $F$. In this case $\mathbf{C}_{T / S}$ contains just the $B$-modes contribution to the power spectrum. Equation (3) requires the signal and the noise to be Gaussian. The gravitational 


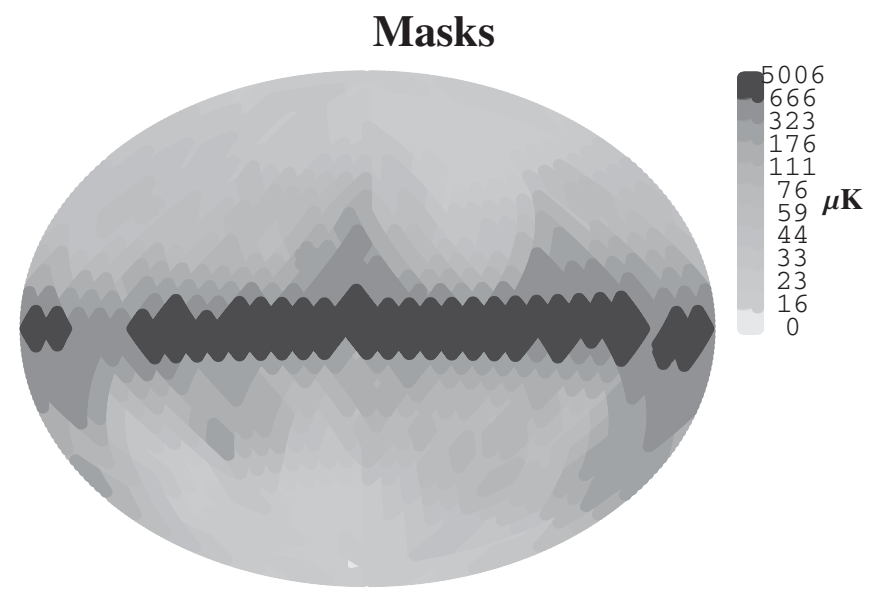

FIG. 1. The masks used for cutting the sky based on HEALPix resolution 3 galactic dust maps in Aitoff projection. Various levels of gray show the way the masks were built by adding areas with higher dust temperature. The units are of black body temperature.

wave signal is Gaussian because it comes from the fluctuations of a linear quantum oscillator. Instrument noise is typically Gaussian due to the central limit theorem; it is assumed here for simplicity that it is white but this assumption may be inappropriate for some experiments.

The Fisher matrix error $\triangle\left(\frac{T}{S}\right)$ is the $1 \sigma$ standard deviation of $\widehat{T / S}$ assuming that $T / S=0$. The caveats of this method are that the error distribution of $\triangle\left(\frac{T}{S}\right)$ is nonGaussian and it depends on $T / S$ (in particular if $T / S$ is increased, the error goes up). During our calculations, we used the galactic dust temperature map [19] to create the masks for our patches. For all the resolutions considered in our calculations, we used the same dust map, sampled at a lower resolution. As we included a higher portion of the sky in our calculations, we kept adding regions contaminated more and more by the foregrounds in our map. They are based on masks, some of which are shown in Fig. 1. For one of the calculations, in order to point out the effect of having a shorter length for the boundary of the cut, we used cuts parallel to the galactic plane.

\section{NUMERICAL METHOD}

We have developed two different numerical methods for evaluating Eq. (3), both of them having as input the scalar and tensor modes generated by CMBFAST [20] with the RECFAST recombination routines [21,22]. We are using maps of various resolutions in the HEALPix [23,24] pixelization. Given the resolution $r$, the number of pixels a HEALPix map has is $N=12\left(4^{r}\right)$. The pixel area is $A=$ $4 \pi / N$, and the side of the pixel is

$$
d=\sqrt{A}=\sqrt{\frac{4 \pi}{N}} .
$$

The Nyquist mode will be

$$
l_{\mathrm{Ny}}=\frac{1}{2} \frac{2 \pi}{d}=\frac{\sqrt{\pi N}}{2} .
$$

We are using both the scalar and the tensor modes with $2 \leq$ $l \leq l_{\mathrm{Ny}} . l_{\mathrm{Ny}}$, and we apply a Gaussian window on them of the form

$$
\mathrm{w}=\exp \left[\frac{-\pi l(l+1)}{3 \alpha N}\right]
$$

here $\alpha$ is a Gaussian window factor. To avoid high- $l E$ modes leaking down to low $l$ through pixelization aliasing effects, we require that $\mathrm{w}_{l_{\mathrm{Ny}}}^{E E} \ll C^{\text {noise }}$, which setting $C^{\text {noise }} /\left(\mathrm{w} C_{l_{\mathrm{Ny}}}^{E E}\right)=10$ gives

$$
\alpha=\frac{\pi^{2}}{12\left(\ln \frac{C_{\mathrm{Ny}}^{E E}}{C^{\text {noise }}}+\ln (10)\right)} ;
$$

and in Fig. 2 we can see the window function satisfying the above requirements applied to the $E$ and $B$ modes.

The exact method uses the convolution algorithm of Ref. [25] to generate the $\mathbf{C}_{0}$ matrix starting from $C_{l}^{E E}$, $C^{\text {noise }}$ and to implement Eq. (2) for the $\mathbf{C}_{T / S}$ matrix from $C_{l}^{B B}$. After generating the matrices, we want to compute $\frac{1}{2} \operatorname{Tr}\left[\left(\mathbf{C}_{0}^{-1} \mathbf{C}_{T / S}\right)^{2}\right]$. We use the Cholesky method for computing the inverse of $\mathbf{C}_{0}$ and then the back substitution method for the multiplication with $\mathbf{C}_{T / S}$. The final step is taking the square of the final matrix, which is done classically. As we want to evaluate the same quantities for different cuts of the sky, we can save processor time by doing the computation progressively increasing the area which is covered. The computer memory is another limiting factor for our computation. Given that $N$ is the number of pixels, this method needs to store in the memory $3 N^{2} / 4$ double precision numbers, to the leading order of $N$. With 4 GB of memory available, $r=5$ is the highest resolution

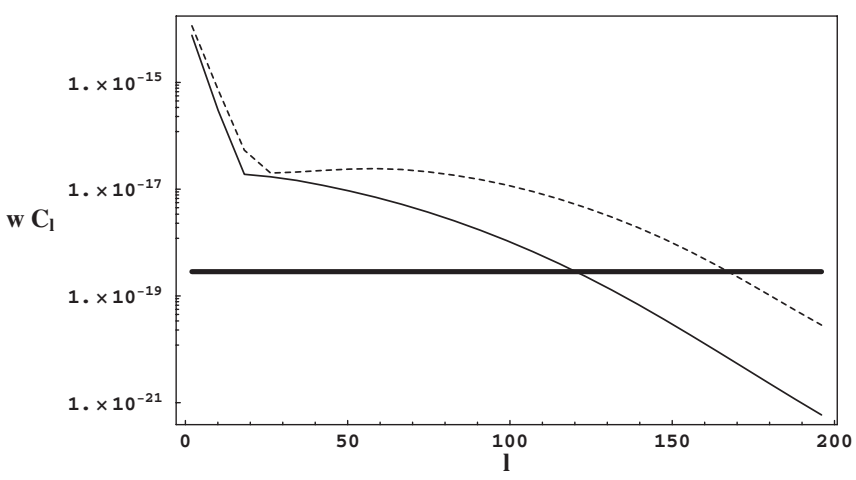

FIG. 2. $\mathrm{w} C_{l}^{E E}, \mathrm{w} C_{l}^{B B}$, and the lensing + detector noise level after applying the Gaussian window for $T / S \simeq 1 / 2$. ${ } C_{l}^{E E}$ is represented by the dashed line, $\mathrm{w}_{l}^{B B}$ by the continuous line, and the lensing + detector noise level by the thick horizontal line. These are the values used for resolution 6 , where $l_{\mathrm{Ny}}=196$. 
at which we can perform the exact calculation. The exact method is an $N^{3}$ process, which makes the processor time required for higher resolutions to increase rapidly.

In addition to the method described above, we are using a Monte Carlo method to evaluate the Fisher matrix. It evaluates

$$
F_{T / S, T / S}=\frac{1}{2}\left\langle\mathbf{x}^{\dagger} \mathbf{C}_{0}^{-1} \mathbf{C}_{T / S} \mathbf{C}_{0}^{-1} \mathbf{C}_{T / S} \mathbf{x}\right\rangle
$$

where $\mathbf{x}$ is a randomly generated vector and $\mathbf{C}_{0}^{-1} \mathbf{x}$ is computed iteratively, without having to take the inverse of the $\mathbf{C}_{0}$ matrix. We evaluate $\mathbf{C}_{0}^{-1} \mathbf{x}$ using the unpreconditioned conjugate-gradient method with the spherical harmonic transform routines described in Ref. [25]. We compute the mean for different realizations of $\mathbf{x}$ and use the standard deviation to obtain $1 \sigma$ errors. This method is more efficient than the exact approach for resolutions higher than 4 as it scales as $\propto N^{3 / 2}$ (the number of conjugate-gradient iterations needed to solve $\mathbf{C}_{0}^{-1} \mathbf{x}$ and the number of realizations of $\mathbf{x}$ needed to compute the trace are roughly 400 and 25 , respectively, and do not depend significantly on the resolution, but depend more on the number of pixels chosen within the mask and also on the choice of the mask). We chose a value of $5.0 \mu \mathrm{K}$ arcmin for the noise, which is the contribution of the lensing noise level. The results can be scaled down to lower lensing + detector noise if lens cleaning is used [5,26,27], e.g. a $2.5 \mu \mathrm{K}$ arcmin experiment would have an uncertainty $\triangle(T / S)$ that is reduced by a factor of 4 .

\section{RESULTS}

We have computed the Fisher matrix using Eq. (3), and from this, we can calculate

$$
\triangle\left(\frac{T}{S}\right)=F_{T / S, T / S}^{-1 / 2}
$$

We used both methods for resolutions 4 and 5, while for resolution 6, we used only the Monte Carlo method. In order to measure more accurately the tensor-to-scalar ratio, a scientific mission would need to cover a higher fraction of the sky. We make plots showing the uncertainty in the tensor-to-scalar ratio $\left(\triangle\left(\frac{T}{S}\right)\right)$ versus the sky fraction we need to cover in order to achieve this uncertainty. As the Monte Carlo method does not output the exact value of the Fisher matrix, but it only estimates it, every time we used the Monte Carlo method, the points are presented with the corresponding $1 \sigma$ error bars. The area of the sky used in our calculations is chosen based on the masks which were presented in a previous section (Fig. 1).

For the entire sky, the uncertainty in the tensor-to-scalar ratio is about $1.5 \times 10^{-5}$. According to the usual scaling $\triangle\left(\frac{T}{S}\right) \propto f_{\text {sky }}^{-1 / 2}$, for a $70 \%$ coverage of the sky we should obtain $\triangle\left(\frac{T}{S}\right)=1.8 \times 10^{-5}$; instead, from our calculations we get $\triangle\left(\frac{T}{S}\right)=3.2 \times 10^{-5}$, nearly a factor of 2 degradation over the idealized case. For $f_{\text {sky }}>0.7$ the scaling is $f_{\text {sky }}^{-2}$, as it is depicted in Fig. 4 by the straight diagonal line. While the scaling is less steep below, we still find it is steeper than expected. To achieve a $3 \sigma$ detection of $T / S=10^{-3}$ we need $15 \%$ sky coverage, compared to $0.3 \%$ from idealized scaling, while for $T / S=10^{-2}$ the required sky coverage is $1 \%$.

To interpret these results the most relevant question is what constraints do these results place on the required level of foreground subtraction. Typically in CMB we remove some part of the sky where foregrounds are just too difficult to deal with, while in the remaining area some foreground subtraction method is applied to reduce the contamination below the required level. This level of course depends not only on the foregrounds, but also on the expected signal. To get a lower $\triangle\left(\frac{T}{S}\right)$ we have to map a larger fraction of the sky, but this means that we have to get into more contaminated regions of the sky, which requires better methods for cleaning the foreground. The cleaning methods are based on expected frequency scaling of dust radiation, which differs from that of CMB. However, frequency scaling may not be the same at every direction because of a different composition of dust grains. This leads to a decorrelation of dust maps at differing frequencies. This provides a fundamental limitation to foreground cleaning that is very difficult to circumvent. Since we do not have data about the polarization of dust radiation we will just assume different levels for it. We also do not know how decorrelated dust maps are as a function of frequency separation. Rough expectation is that dust is polarized at a few percent level, so we expect that cleaning at $1 \%$ should be relatively straightforward, at $0.1 \%$ significantly more challenging, and at $0.01 \%$ may be impossible. The tensorto-scalar ratio for which the polarized signal is equal to the polarized fraction of the dust thermal emission is defined as:

$$
\frac{T}{S}=\frac{\left(p T_{\text {dust }}\right)^{2}}{\left.\sum_{l=2}^{l_{\max }} \frac{2 l+1}{4 \pi} C_{l}^{B B}\right|_{T / S=1}} ;
$$

where $p$ is the polarized fraction of the dust thermal emission. We will assume we work at $90 \mathrm{GHz}$, where dust signal is relatively low and we ignore the contribution from polarized synchrotron emission, which is expected to be similar to or slightly less than the dust polarization at $90 \mathrm{GHz}$ [28]. Because of this many of the upcoming polarization experiments are expected to operate at higher frequencies where dust signal is stronger relative to CMB, in which case our results may be overly optimistic and need to be rescaled appropriately. Note that our analysis is simplistic in the sense that we only use Eq. (10) to assess the detectability prospects and do not include more detailed information on how the two signals vary with scale. It should nevertheless give a good ballpark estimate of the required levels of decontamination. 


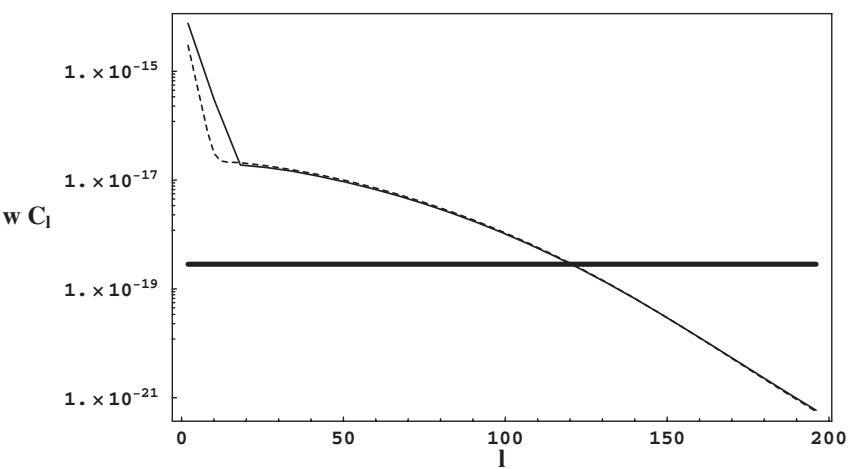

FIG. 3. ${ }^{w} C_{l}^{B B}$ for two different values of the optical depth to the reionization $\tau$. The dashed line corresponds to a value of $\tau=$ 0.07 and the continuous line to $\tau=0.17$. The thick horizontal line is the lensing + detector noise level.

For example, if we want to detect a tensor-to-scalar ratio of $10^{-4}$, we would need to map $70 \%$ of the sky and the polarized fraction of the dust thermal emission needs to be less than $0.01 \%$., while if we want to detect a tensor-toscalar ratio of $10^{-3}$, we need to map $15 \%$ of the sky and the polarized fraction of the uncleaned dust thermal emission in this region cannot exceed $0.1 \%$ (Fig. 5).

Besides parameters that depend on the instrument, there are also cosmological parameters that can influence the detection of $B$ modes. As it was discussed in the Introduction section of the paper, a cosmological parameter which has a large influence on the results of our calculations is the optical depth to the reionization $\tau$. For example, if we vary the value of $\tau$ from 0.17 , to 0.07 , for HEALPix resolution 4 the value of $\triangle\left(\frac{T}{S}\right)$ increases by half an order of magnitude, while the effect of varying the optical depth on the $B$ modes can be seen in Fig. 3. We show $\triangle\left(\frac{T}{S}\right)$ for different values of optical depth $\tau$ in Fig. 6 .

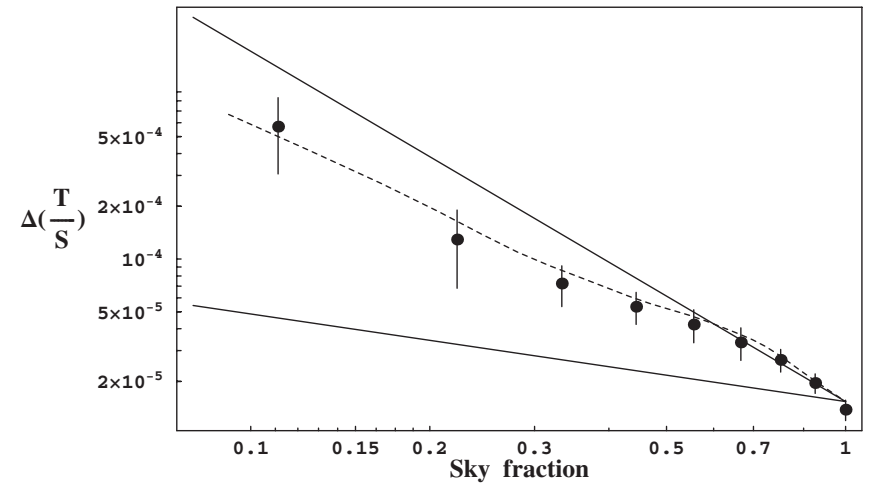

FIG. 4. $\triangle\left(\frac{T}{S}\right)$ as a function of sky fraction. The dashed line is computed based on the exact method for resolution 4, while the points with error bars are based on the Monte Carlo method for resolution 6 . The continuous lines represent the theoretical scaling of $\triangle\left(\frac{T}{S}\right)$. The lower line corresponds to the idealized scaling $\triangle\left(\frac{T}{S}\right) \propto f_{\text {sky }}^{-1 / 2}$, while the upper line is $\triangle\left(\frac{T}{S}\right) \propto f_{\text {sky }}^{-2}$ and fits better the actual results for $f_{\text {sky }}>0.7$.

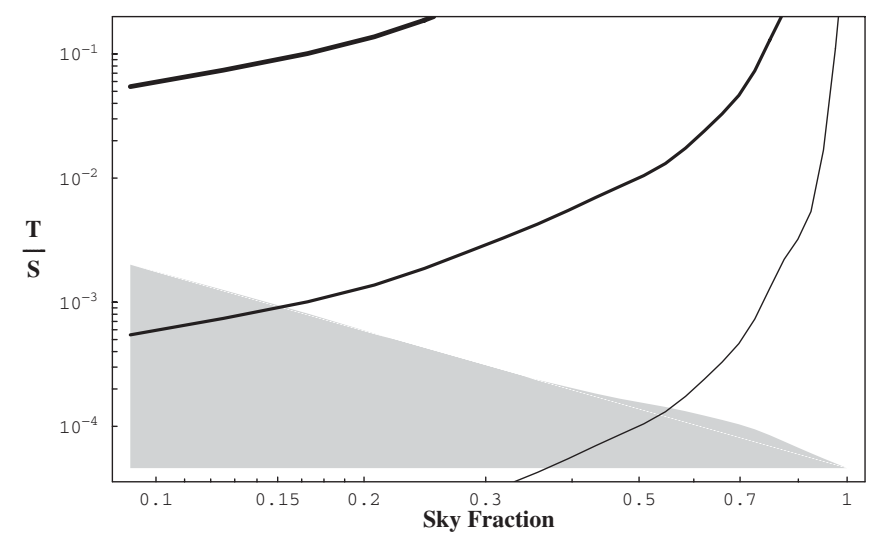

FIG. 5. The lines represent the fraction of the sky for which the polarized fraction of the dust thermal emission is less than the polarized signal, as defined in Eq. (10), assuming the polarized fraction of the dust thermal emission to be $1 \%$ (top), $0.1 \%$ (middle), and $0.01 \%$ (bottom). Dashed area is excluded if we require a detection of tensor-to-scalar ratio $\frac{T}{S}$ at a $3 \sigma$ confidence. For example, to detect $T / S=10^{-3}$ at $3 \sigma$ we need to observe $15 \%$ of the sky and if that is chosen to be the cleanest region of the sky then the dust polarization in this region needs to be cleaned at $0.1 \%$ level of its intensity.

As space missions require a large budget, the scientific community is also considering ground-based or balloonborne experiments. They can achieve a higher angular resolution, but their sky coverage has to suffer. We are also considering this case, by doing a calculation at HEALPix resolution 7 , confined to declinations $\delta<$ $-30^{\circ}$ around the terrestrial south pole. For the purpose of this calculation, we ignored the contribution of the reionizatoin peak to the tensor modes, by considering only $C_{l}^{B B}$ with $l>20$. The results of this calculation can be seen in Fig. 7. Thus, in order to be able to detect $\triangle\left(\frac{T}{S}\right)=$ $10^{-2}$ at $3 \sigma$, we need to look at $1 \%$ of the sky and from Eq. (10) we find that the polarized dust signal must be cleaned down to $1 \%$ of its intensity.

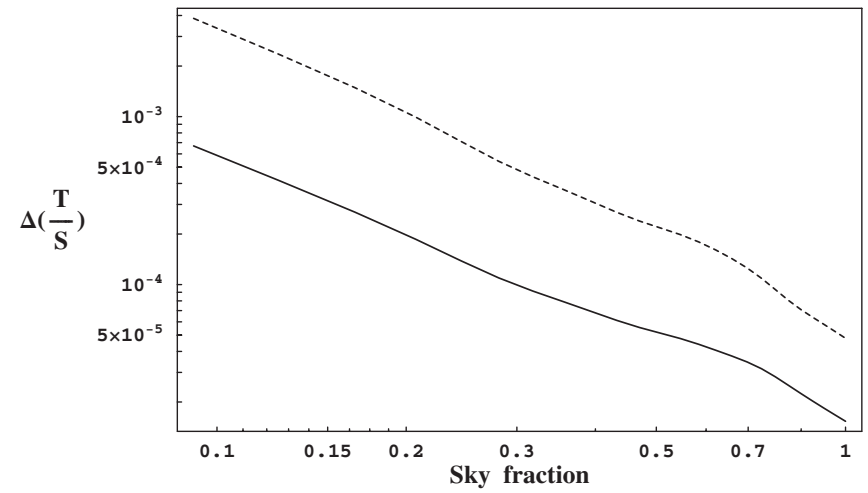

FIG. 6. $\triangle\left(\frac{T}{S}\right)$ computed based on the exact method for resolution 4 . The dashed line corresponds to an optical depth $\tau=0.07$, while the continuous line corresponds to $\tau=0.17$. 


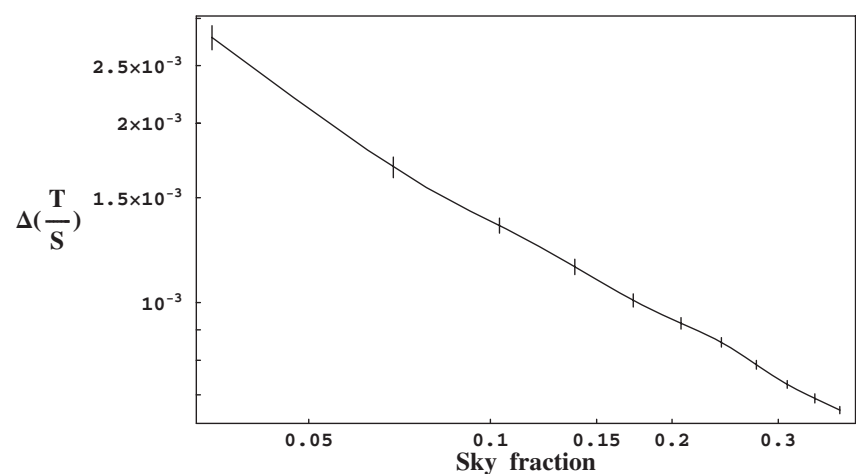

FIG. 7. $\triangle\left(\frac{T}{S}\right)$ for HEALPix resolution 7 for a map confined to Earth's southern hemisphere, declinations $\delta<-30^{\circ}$. The graph is obtained by ignoring the reionization peak for the $B$ modes and using the Monte Carlo method, with $1 \sigma$ error bars shown on the graph. The continuous line is a cubic spline through the considered points.

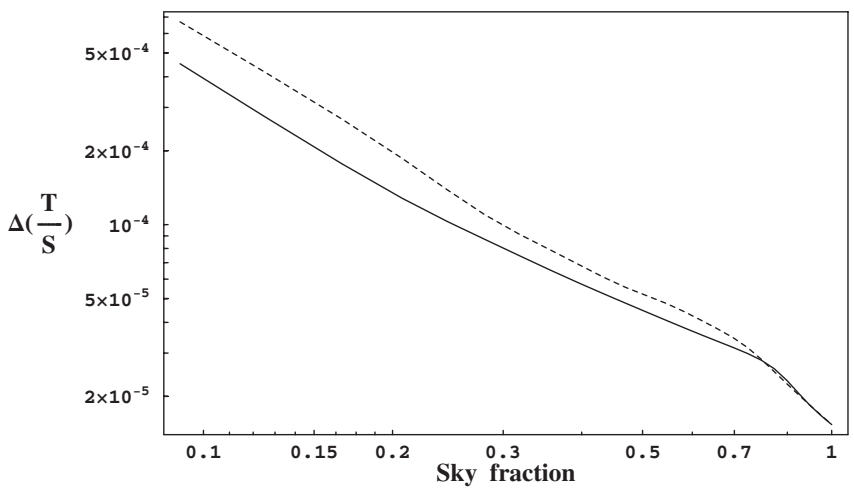

FIG. 8. The difference in $\triangle\left(\frac{T}{S}\right)$ for two different types of cuts. The continuous line is obtained for the parallel equatorial cut. Both results are obtained through the exact method for HEALPix resolution 4.

\section{DISCUSSION AND CONCLUSIONS}

The main conclusion we get from these results is that the Fisher matrix element $F_{T / S, T / S}$ does not depend linearly on the sky percentage considered in the map. Instead, it depends more like $F_{T / S, T / S} \propto f_{\text {sky. }}^{4}$. This is in some way expected as the harmonic decomposition of the sky is nonlocal and cutting off some parts of the sky affects the rest of it also especially for the large scale where the $B$ modes are more important. It is not an exact power law dependence, but as it can be seen from Fig. 4, the power law could be a good approximation. We fitted the points from $10 \%$ to $100 \%$ in sky fraction to a power law. There is a disagreement between this dependence and the one from Fig. 8 of Ref. [10], where $F_{T / S, T / S} \propto f_{\text {sky }}^{1.7}$. The method proposed in Ref. [10] uses projected $E$ and $B$ modes, which triggers loss of information in the low multipoles. In the pixel space, this loss increases for small sky patches. To give an idea of the way low multipoles affect our analysis, in the case where the effect of the $B$ modes in the reionization peak was not considered (Fig. 7), $F_{T / S, T / S} \propto f_{\text {sky }}^{1.2}$. We considered different pixelizations by changing the resolution of our HEALPix maps and we can see from Fig. 4 that this does not affect much the noise levels, leaving the results unchanged. The way we choose to make the cut is mildly important in evaluating the results. If instead of using a cut based on the galaxy dust maps, we take a straight symmetric cut, parallel to the galactic equator, we get higher $F_{T / S, T / S}$ values, but the change is modest (Fig. 8).

Our results suggest that to achieve a detection of $T / S$ at $10^{-3}$ level one needs to observe $15 \%$ of the whole sky as opposed to $0.3 \%$ naively expected. This makes the case for a space mission stronger if such levels of $T / S$ can be motivated theoretically. However, this is somewhat dependent on the assumed optical depth, which we do not know yet with enough precision. Moreover, it is unclear if we can actually foreground clean the observed portion of the sky sufficiently well; as we have indicated the required levels are challenging indeed, in the case of $T / S=10^{-3}$ they require a factor of 10-100 reduction of polarized dust emission even if the cleanest part of the sky is chosen, agreeing with the results from [13], where the authors considered several specific situations. It is premature to give a precise verdict in favor of a space mission or a ground-based/balloon-borne experiment, what is clear is that we need more data from the experiments currently running in order to put a better constraint on the parameters that influence the detection of $B$ modes, and then decide for the type of experiment we have to build next.
[1] U. Seljak, Astrophys. J. 482, 6 (1997).

[2] M. Zaldarriaga and U. Seljak, Phys. Rev. D 55, 1830 (1997).

[3] M. Kamionkowski, A. Kosowsky, and A. Stebbins, Phys. Rev. D 55, 7368 (1997).

[4] M. Kamionkowski and A. Kosowsky, Phys. Rev. D 57, 685 (1998).
[5] U. Seljak and C. M. Hirata, Phys. Rev. D 69, 043005 (2004).

[6] A. Lazarian and D. Finkbeiner, New Astron. Rev. 47, 1107 (2003).

[7] A. de Oliveira-Costa, M. Tegmark, C. O'dell, B. Keating, P. Timbie, G. Efstathiou, and G. Smoot, New Astron. Rev. 47, 1117 (2003). 
[8] A. Slosar, U. Seljak, and A. Makarov, Phys. Rev. D 69, 123003 (2004).

[9] A. Slosar and U. Seljak, Phys. Rev. D 70, 083002 (2004).

[10] A. Lewis, A. Challinor, and N. Turok, Phys. Rev. D 65, 023505 (2002).

[11] E.F. Bunn, M. Zaldarriaga, M. Tegmark, and A. de Oliveira-Costa, Phys. Rev. D 67, 023501 (2003).

[12] C. M. Hirata, A. Loeb, and N. Afshordi, Phys. Rev. D 71, 063531 (2005).

[13] L. Verde, H. Peiris, and R. Jimenez, astro-ph/0506036.

[14] M. Zaldarriaga, Phys. Rev. D 55, 1822 (1997).

[15] C. L. Bennett, M. Halpern, G. Hinshaw, N. Jarosik, A. Kogut, M. Limon, S. S. Meyer, L. Page, D. N. Spergel, G. S. Tucker et al., Astrophys. J. Suppl. Ser. 148, 1 (2003).

[16] A. Kogut, D. N. Spergel, C. Barnes, C. L. Bennett, M. Halpern, G. Hinshaw, N. Jarosik, M. Limon, S. S. Meyer, L. Page et al., Astrophys. J. Suppl. Ser. 148, 161 (2003).

[17] D. N. Spergel, L. Verde, H. V. Peiris, E. Komatsu, M. R. Nolta, C. L. Bennett, M. Halpern, G. Hinshaw, N. Jarosik, A. Kogut et al., Astrophys. J. Suppl. Ser. 148, 175 (2003).

[18] U. Seljak, A. Makarov, P. McDonald, S. F. Anderson, N. A. Bahcall, J. Brinkmann, S. Burles, R. Cen, M. Doi, J. E. Gunn et al., Phys. Rev. D 71, 103515 (2005).

[19] D. P. Finkbeiner, M. Davis, and D. J. Schlegel, Astrophys.
J. 524, 867 (1999).

[20] U. Seljak and M. Zaldarriaga, Astrophys. J. 469, 437 (1996).

[21] S. Seager, D. D. Sasselov, and D. Scott, Astrophys. J. Lett. 523, L1 (1999).

[22] S. Seager, D. D. Sasselov, and D. Scott, Astrophys. J. Suppl. Ser. 128, 407 (2000).

[23] K. M. Górski et al., in Evolution of Large Scale Structure: From Recombination to Garching, edited by A. J. Banday, R. S. Sheth, and L. Da Costa (PrintPartners, Ipskamp, NL, 1999), p. 37.

[24] K. M. Górski, E. Hivon, A. J. Banday, B. D. Wandelt, F. K. Hansen, M. Reinecke, and M. Bartelmann, Astrophys. J. 622, 759 (2005).

[25] C. M. Hirata, N. Padmanabhan, U. Seljak, D. Schlegel, and J. Brinkmann, Phys. Rev. D 70, 103501 (2004).

[26] L. Knox and Y.S. Song, Phys. Rev. Lett. 89, 011303 (2002).

[27] M. Kesden, A. Cooray, and M. Kamionkowski, Phys. Rev. Lett. 89, 011304 (2002).

[28] E. Carretti, D. McConnell, N. M. McClure-Griffiths, G. Bernardi, S. Cortiglioni, and S. Poppi, Mon. Not. R. Astron. Soc. 360, L10 (2005). 